\title{
Measurement of Insall Salvati ratio and modified Insall Salvati ratio to assess the position of the patella in South Indian population
}

\author{
Arun Kumar C.*, Ganesan G. Ram \\ Department of Orthopaedics, Sri Ramachandra University, Porur, Chennai, India \\ Received: 24 November 2016 \\ Revised: 10 December 2016 \\ Accepted: 12 December 2016 \\ *Correspondence: \\ Dr. Arun Kumar C., \\ E-mail: drarunc16@gmail.com \\ Copyright: $@$ the author(s), publisher and licensee Medip Academy. This is an open-access article distributed under \\ the terms of the Creative Commons Attribution Non-Commercial License, which permits unrestricted non-commercial \\ use, distribution, and reproduction in any medium, provided the original work is properly cited.
}

\begin{abstract}
Background: This is an observational study that was carried out to find the mean Insall Salvati ratio and modified Insall Salvati ratio in people coming to outpatient department requiring radiograph of knee joint. .

Methods: Radiological assessment of 200 knees which included 100 men and 100 women above the age group of 18,in a period of 6 months for which Insall Salvati and modified Insall Salvati ratio were calculated.

Results: The mean Insall Salvati ratio was calculated to be 0.805 and the mean modified Insall Salvati ratio was 0.605 in South Indian.

Conclusions: Based on the above study i conclude that majority of South Indian population had patella baja with female predominance.
\end{abstract}

Keywords: Insall Salvati ratio, Modified Insall Salvati ratio, Patella baja

\section{INTRODUCTION}

The integral component of the extensor mechanism of the knee joint is the patella. Quadriceps muscle efficiency can be increased by increasing the leverage of the patella. $^{1,2}$ Throughout the range of motion patella increases the extension moment $\mathrm{arm}^{3}$ Insall Salvati ratio is the ratio between the length of the patellar tendon (LT) over the length of the patella (LP). Whereas modified Insall Salvati ratio is measured slightly differently by measuring the distance from the inferior margin of the patellar articular surface (as opposed to the lower pole of the patella itself) to the patellar tendon insertion and length of the patellar articular surface. ${ }^{4}$

The above parameters describe whether it is high riding patella (patella alta) or low riding patella (patella baja). A high riding patella may be associated with recurrent lateral dislocation or subluxation of patella, chondromalacia of patella, and Sinding-Larsen-Johansson disease and patellar and quadriceps tendonitis and OsgoodSchlatter disease..$^{5-7}$ A low-riding patella (patella baja) may be associated with quadriceps tendon rupture, neuromuscular disorders, achondroplasia, and postoperative advancement of the tibial tuberosity. ${ }^{8-10}$

\section{METHODS}

This study included 200 people seen on a OP basis in Sri Ramachandra medical college and research institute between January 2016 to June 2016, chosen randomly, splitting up the study population by 100 males and 100 females to avoid gender based bias with no previous history of trauma, surgeries or any pathologies in and around the knee joint. Patients with bilateral knee pathology and people below the age of 18 were excluded from the study. Radiographic evaluation using x-rays of the knee joint- lateral view with knee kept in 300 flexion. From aforementioned radiographs Insall Salvati ratio and modified Insall Salvati ratio were calculated. 


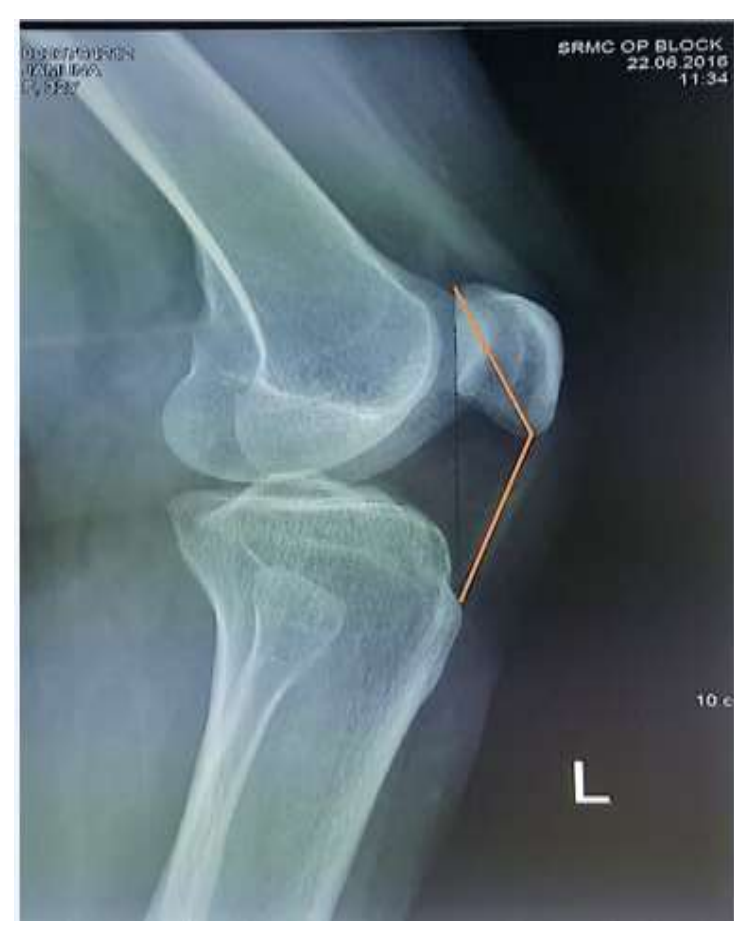

Figure 1: Insall Salvati ratio.

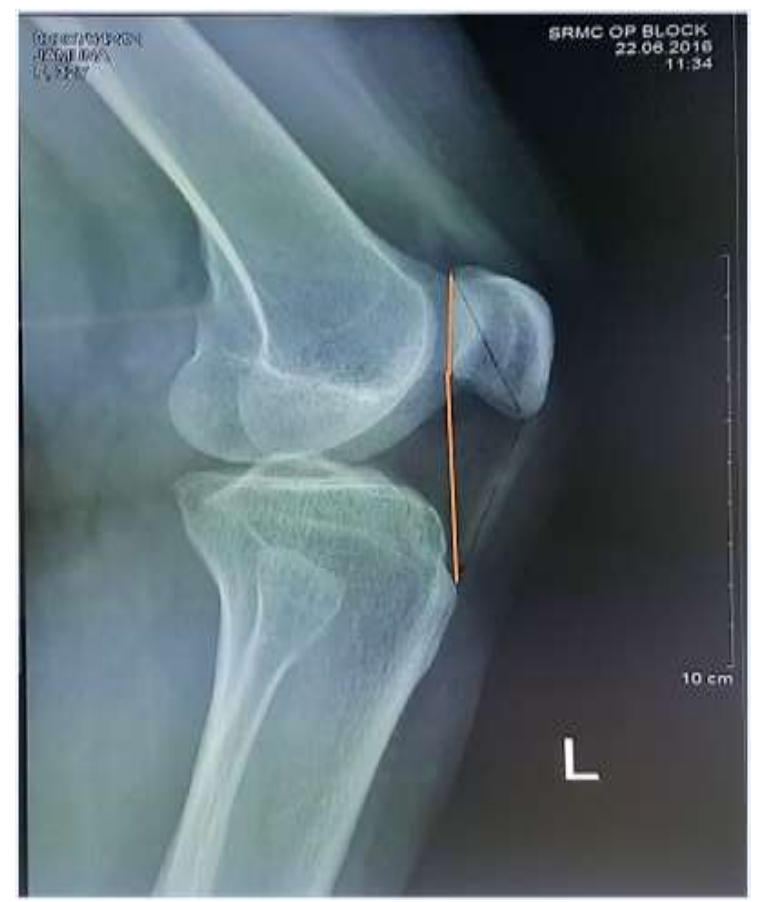

Figure 2: Modified Insall Salvati ratio.

\section{RESULTS}

The mean Insall Salvati ratio for 100 males were found to be 1.41 and for 100 females were found to be 1.28 . The mean modified Insall Salvati ratio for 100 males were 1.81 and for 100 females were 1.75. The mean Insallsalvati ratio for 200 south Indian population were found to be 1.34 and mean modified Insall Salvati ratio for 200 south Indian population was found to be 1.78 .
Table 1: Mean Insall Salvati and modified Insall Salvati ratio values.

\begin{tabular}{|lll|}
\hline Sample & $\begin{array}{l}\text { Insall Salvati } \\
\text { ratio }\end{array}$ & $\begin{array}{l}\text { Modified Insall } \\
\text { Salvati ratio }\end{array}$ \\
\hline Male & 1.41 & 1.81 \\
\hline Female & 1.28 & 1.75 \\
\hline Mean & 1.34 & 1.78 \\
\hline
\end{tabular}

\section{DISCUSSION}

From the above study, it can be concluded that there is no much of a difference between the mean modified Insall Salvati ratio between the male and female being 1.81 and 1.75 respectively. Patella alta and patella baja represent the 2 ends of the scale of the LT/LP ratio. The Insall Salvati ratio is easy to calculate and independent of the degree of knee flexion. ${ }^{4}$ The normal ratio is $1.02 \pm 0.13$; values $>1.2$ are suggestive of patella alta and values $<0.8$ are suggestive of patella baja. ${ }^{8,9}$

Anomalies in patella position are associated with derangements of patellofemoralfunction such as chondromalacia and recurrent subluxation or dislocation of the patella. ${ }^{5,11-14}$ In our study the mean Insall Salvati ratio appears to be towards patellar alta. Similarly, in the study done by Upadhyay et al showed the mean Insall Salvati ratio for people in central India to be 1.14 whereas in our study which involved south Indian population showed 1.34. Squatting and sitting crosslegged are common activities of daily living in Indian cultures. The Insall Salvati ratio is often restricted to chair-sitting western populations and may not be generalised to others. ${ }^{15}$

In a southern Chinese population, the position of patella is 15 to $20 \%$ higher than that in western populations. A patella alta index of $>3.4$ is considered abnormal in these population. ${ }^{16}$ The patellar position is empirical for evaluation of the anatomic alignment of the knee and anterior knee pain. ${ }^{17,18}$ A patella alta may cause pain secondary to patellar instability or chondromalacia patellae that results from abnormal patellofemoral junction contact stress leading to arthritis. ${ }^{19}$

The use of the Insall Salvati ratio to determine the patellar position is less applicable to Indian populations in which squatting, sitting cross-legged, and kneeling are customs. We propose that the normal range of the ratio for squatters among Indian populations be 0.7 to $1.5{ }^{15}$

\section{CONCLUSION}

Though the results may show the spectrum of Insall Salvati ratio towards patella alta when compared to western people it can be attributed to sitting cross legged, squatting and kneeling customs of south Indian population. Modified Insall Salvati ratio could be used to assess the contact stress at patellofemoral junction, it can be used as a predictor for arthritis. 
Funding: No funding sources

Conflict of interest: None declared

Ethical approval: The study was approved by the institutional ethics committee

\section{REFERENCES}

1. Hohl M. Fractures of the patella. In: Fractures. Rockwood CA Jr, Green DP, editors. Philadelphia: JB Lippincott; 1975: 1148-1156.

2. Perry J, Antonelli D, Ford W. Analysis of knee-joint forces during flexed-knee stance. J Bone Joint Surg Am. 1975;57:961-7.

3. Kaufer H. Mechanical function of the patella. J Bone Joint Surg Am. 1971;53:1551-60.

4. Grelsamer RP, Meadows S. The modified InsallSalvati ratio for assessment of patellar height. Clin Orthop Relat Res. 1992;282:170-6.

5. Andersen PT. Congenital deformities of the knee joint in dislocation of the patella and achondroplasia. Acta Orthop Scand. 1958;28:27-50.

6. Smillie IS. Injuries of the knee joint. 4th edition. Edinburgh: Livingstone; 1970.

7. Medlar RC, Lyne ED. Sinding-Larsen-Johansson disease. Its etiology and natural history. J Bone Joint Surg Am. 1978;60:1113-6.

8. Noyes FR, Wojtys EM, Marshall MT. The early diagnosis and treatment of developmental patella infera syndrome. Clin Orthop Relat Res. 1991;265:241-52.

9. Tria AJ Jr, Alicea JA, Cody RP. Patella baja in anterior crutiate ligament reconstruction of the knee. Clin Orthop Relat Res. 1994;299:229-34.

10. Kalichman L, Zhang Y, Niu J, Goggins J, Gale D, Felson DT, et al. The association between patellar alignment and patellofemoral joint osteoarthritis features - an MRI study. Rheumatology (Oxford). 2007;46:1303-8.

11. Lancourt JE, Cristini JA. Patella alta and patella infera. Their etiological role in patellar dislocation, chondromalacia, and apophysitis of the tibial tubercle. J Bone Joint Surg Am. 1975;57:1112-5.

12. Insall J, Falvo KA, Wise DW. Chondromalacia patellae.A prospective study. J Bone Joint Surg Am. 1976;58:1-8.

13. Karadimas JE, Piscopakis N, Syrmalis L. Patella alta and chondromalacia. IntOrthop. 1981;5:247-9.

14. Bentley G, Dowd G. Current concepts of etiology and treatment of chondromalacia patellae. Clin Orthop Relat Res. 1984;189:209-28.

15. Upadhyay S, Raza HKT, Srivastava P. Position of the patella in adults in central India: evaluation of the Insall-Salvati ratio. J Ortho. Surg. 2013;21(1):23-7.

16. Leung YF, Wai YL, Leung YC. Patella alta in southern China. A new method of measurement. Int Orthop. 1996;20:305-10.

17. Luyckx T, Didden K, Vandenneucker H, Labey L, Innocenti B, Bellemans J. Is there a biomechanical explanation for anterior knee pain in patients with patella alta? Influence of patellar height on patellofemoral contact force, contact area and contact pressure. J Bone Joint Surg $\mathrm{Br}$. 2009;91:344-50.

18. Metin Cubuk S, Sindel M, Karaali K, Arslan AG, Akyildiz F, Ozkan O. Tibial tubercle position and patellar height as indicators of malalignment in women with anterior knee pain. Clin Anat. 2000;13:199-203.

19. Kannus PA. Long patellar tendon: radiographic sign of patellofemoral pain syndrome-a prospective study. Radiology. 1992;185:859-63.

Cite this article as: Arun Kumar C, Ram GG. Measurement of Insall Salvati ratio and modified Insall Salvati ratio to assess the position of the patella in South Indian population. Int J Res Orthop2017;3:23-5. 